\title{
Políticas de salud bucal en México: Disminuir las principales enfermedades. Una descripción.
}

Revisión

Carlo E. Medina-Solis ${ }^{1,2}$, Gerardo Maupomé ${ }^{3}$, Leticia Avila-Burgos², Ricardo Pérez-Núñez ${ }^{2}$, Blanca Pelcastre-Villafuerte², América P. Pontigo-Loyola ${ }^{1}$.

${ }^{1}$ Área Académica de Odontología, Instituto de Ciencias de la Salud, Universidad Autónoma del Estado de Hidalgo, México.

${ }^{2}$ Centro de Investigación en Sistemas de Salud, Instituto Nacional de Salud Pública, Cuernavaca, Morelos, México.

${ }^{3}$ Oral Health Research Institute, Indiana University / Purdue University at Indianapolis School of Dentistry. Indianapolis, Indiana, USA.

\section{RESUMEN.}

Se presenta una breve descripción de lo que ha sido hasta ahora la historia de las políticas de salud bucal en México. Este trabajo no intenta ser exhaustivo, ni mucho menos un estudio sobre evaluación de políticas de salud bucal, pues se vería enfrentado a la fuerte limitación de la disponibilidad de información desarrollada en el área de la salud bucal. En primer lugar se abordan los inicios de la profesión dental y la forma en la que se desarrolló; su comienzo como una profesión dirigida al individuo y su posterior inmersión en el campo de la Salud Pública. Posteriormente se presentan datos de estudios epidemiológicos realizados en los últimos años, principalmente sobre caries dental en escolares. Termina esta parte tomando en cuenta la definición de salud de la Organización Mundial de la Salud (OMS),

\footnotetext{
* Trabajo presentado durante el Seminario de Métodos Avanzados de Investigación en Sistemas de Salud en el Instituto Nacional de Salud Pública en 2004.
}

y se trata la importancia de la salud bucal como un elemento de la salud general y por ende del bienestar y de la calidad de vida del individuo. Más adelante se describe la política de salud bucal mundial, como marco de referencia para la política de salud en el país. Con relación a México, describimos cómo a través de los años esta política ha planeado e instaurado estrategias tendientes a lograr una mejora en la salud bucal de la población. Concluimos el trabajo con un análisis acerca de cómo ha impactado la política de salud bucal a la salud de ciertos grupos poblacionales. Se propone generar información epidemiológica confiable para poder hacer una evaluación de esta política, con una metodología estricta y adecuada.

(Rev Biomed 2006; 17:269-286)

Palabras clave: salud bucal, epidemiología, caries, fluoruro.

Solicitud de sobretiros: M en C. Carlo E. Medina-Solís. Privada de Altillo s/n entre Av. Central y Pedro Moreno. Colonia San José. C.P. 24040. Campeche, Campeche, México. Tel y Fax: 01 (981) $8110215 . \quad$ Correo Electrónico: cemedinas@yahoo.com Recibido el 7/Abril/2006. Aceptado para publicación el 13/Julio/2006.

Este artículo está disponible en http://www.uady.mx/sitios/biomedic/revbiomed/pdf/rb061745.pdf

Vol. 17/No. 4/Octubre-Diciembre, 2006 


\section{CE Medina-Solis, G Maupomé, L Avila-Burgos, R Pérez-Núñez, B Pelcastre-Villafuerte, y col.}

\section{SUMMARY.}

Oral health policy in Mexico: reducing the principal diseases. A description.

A brief description of what has been the history of the oral health policies in Mexico up to now is presented. This work is not exhaustive, nor is it a study on the evaluation of oral health policy, since if this were the case one would find a lack of information in the field of oral health. Firstly, the origins of the dental profession and the form in which it developed are discussed; its beginnings directed to the individual and its subsequent immersion in the sphere of Public Health are described. Subsequently, data from epidemiological studies carried out in recent years, mainly on dental decay in scholars are presented. The definition of health according to the World Health Organization (WHO), includes the importance of oral health as an element of general health and, therefore, of the welfare and of the quality of life of the individual. Subsequently, world oral health policy, as a reference frame for oral health, is presented. In relation to Mexico, we describe how this police has been planned and established through the years in an effort to improve the oral health of the population. We conclude with an analysis on how this policy on oral health has had an impact in the general health of certain population groups. It is proposed to generate reliable epidemiological information to be able to evaluate this police, following an adequate and strict methodology.

(Rev Biomed 2006; 17:269-286)

Key words: oral health, epidemiology, caries, fluoride.

\section{INTRODUCCIÓN.}

En México, la evolución del perfil epidemiológico muestra un evidente proceso de transición, caracterizado por la coexistencia de enfermedades y muertes por causas infecciosas, con aquellas relacionadas a padecimientos crónico-degenerativos, accidentes, y lesiones. Las enfermedades bucodentales no son la excepción a estas tendencias. Las prioridades a las que se aboca la política de salud bucal en México son disminuir la experiencia de caries dental, las periodontopatías y el cáncer bucal, para lo cual se han diseñado diversos componentes de la política, como son: el educativo preventivo en escolares, el educativo asistencial y el de fluoruración de la sal (1).

Es bien sabido que las enfermedades bucales como la caries dental y las enfermedades periodontales son de las mayor incidencia y prevalencia alrededor del mundo y se encuentran concentradas principalmente en los grupos menos favorecidos, lo que las constituye como problemas de salud pública bucal. Una de las principales tareas de los planeadores en salud es encontrar estrategias para prevenir o controlar estos problemas.

Las investigaciones reportan que durante las últimas décadas la prevalencia y experiencia de caries han declinado en algunos segmentos de la población de la mayoría de los países desarrollados (2-8) y que esta tendencia se ha visto parcialmente reflejada en algunos países de América Latina y el Caribe $(9,10)$.

También, que la causa más probable de la disminución en la prevalencia de caries en el mundo es la instauración de programas de educación, prevención y tratamiento de esta enfermedad $(2,3)$, cambios en los criterios diagnósticos $(11,12)$ y la introducción de fluoruros en sus diversas presentaciones (13-16). Sin embargo, otros estudios reconocen que no sólo el fluoruro tiene influencia en la disminución de caries y que otros factores se deben considerar $(11,17,18)$. Esta diversidad de factores es fundamental para entender la relación entre las características del perfil de salud en una población, y las políticas de salud imperantes en ese ambiente.

Es generalmente aceptado que un número amplio de factores determinan la salud de la población. Un modelo propuesto por Lalonde (19) (figura 1) muestra que existen cinco grupos

\section{Revista Biomédica}


Políticas de salud bucal en México

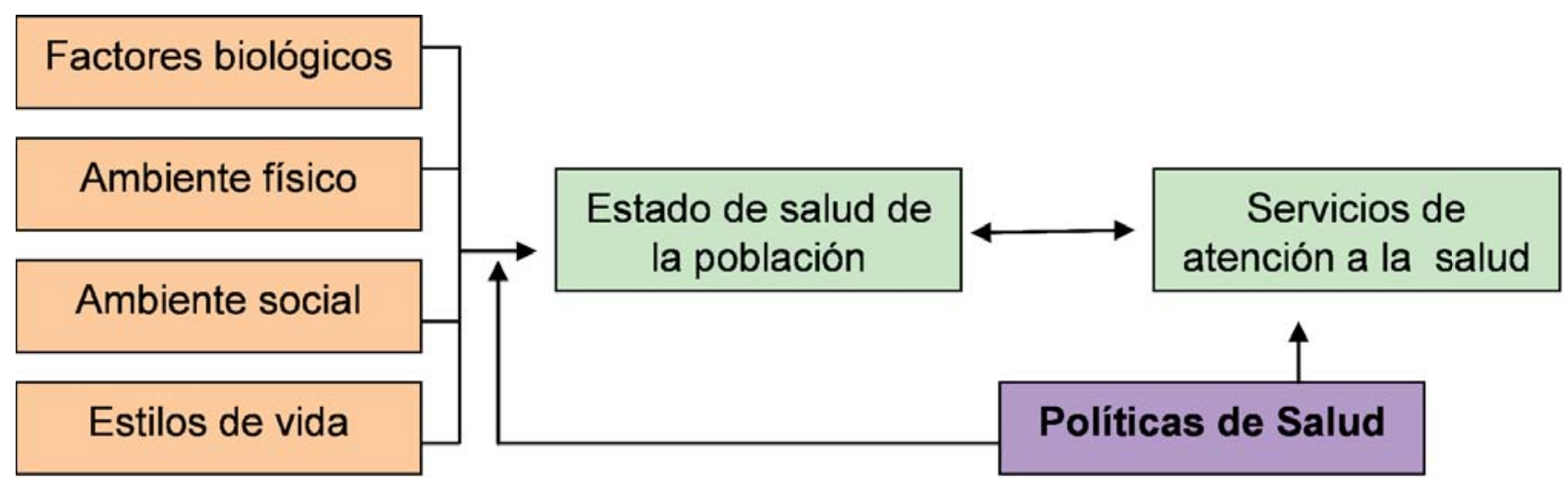

Figura 1.- Factores que afectan la salud de la población según Modelo Lalonde.

Fuente: Lalonde M. A new perspective on the health of the Canadians: a working document. Ottawa:

Government of Canada, 1974.

de variables que determinan la salud. Entre las variables de este modelo se encuentran las políticas de salud (20).

A nivel nacional, la Secretaría de Salud es la responsable directa de los servicios de salud y de la educación en salud en todas sus áreas. Sin embargo, hay varios factores que quedan fuera de su alcance; como ejemplo tenemos las decisiones a nivel individual.

Un aspecto importante para lograr la prevención y control de las enfermedades bucales ha sido la instauración de políticas en salud bucal. México, como país miembro de organismos internacionales como la Organización Mundial de la Salud (OMS) y la Organización Panamericana de la Salud (OPS), se propone cumplir las metas establecidas por estos organismos (figura 2).
El objetivo de este trabajo es revisar y describir la evolución de las políticas en salud bucal instauradas en México, así como evaluar indirectamente su impacto en la salud bucal de la población.

\section{Antecedentes.}

La salud bucal en México ha atravesado por distintas etapas en su historia y continúa actualmente su desarrollo. Desde la época prehispánica los pobladores de diversas culturas asentadas en el territorio nacional ya realizaban distintos tratamientos, como desgastes selectivos e incrustaciones sobre sus dientes. Se empleaban diversas plantas en la terapéutica dental, principalmente para mitigar los dolores que provocaban la caries dental y las parodontopatías.

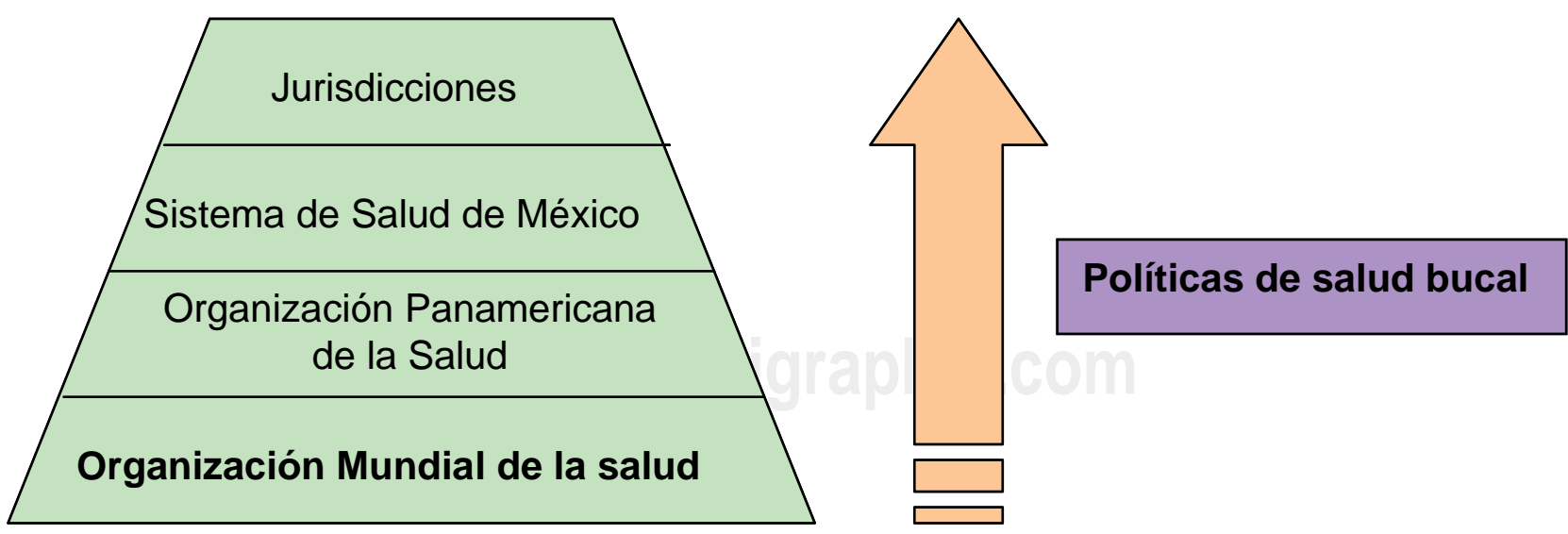

Figura 2.- Dirección de las políticas y metas de salud que van de lo macro a lo micro. 
CE Medina-Solis, G Maupomé, L Avila-Burgos, R Pérez-Núñez, B Pelcastre-Villafuerte, y col.

Durante la época colonial e independiente (15301900), la odontología fue practicada a nivel individual curativo y restaurativo más que a nivel colectivo, sin la necesidad de un título profesional para ejercerla, lo cual cambió a principios del siglo XX (21-24). La enseñanza universitaria odontológica y la práctica dental clínica cumplieron su primer centenario como las actividades orientadas a atender la salud de la sociedad mexicana, ya que es a principios del siglo XX cuando comenzó oficialmente la profesión odontológica en México. En esa centuria la profesión dental ha sido poseedora de gran prestigio. La sociedad ha financiado de forma continua la preparación de estos especialistas: la Facultad de Odontología de la UNAM, escuela universitaria más grande del país, gradúa de 600 a 800 dentistas cada año. La planeación que ha prevalecido supone que cuanto mayor es el número de personal clínico, la demanda es enfrentada más efectivamente.

En 1902 se publicó en el diario oficial de la federación el plan de estudios para la carrera de Cirujano Dentista, la cual era cursada en el Consultorio Nacional de Enseñanza Dental. Posteriormente, en 1911 se creó la Escuela Nacional de Enseñanza Dental. En 1942 se fundó la Asociación Dental Mexicana. El énfasis de la práctica dental sigue siendo a nivel individual, primordialmente limitándose a los tratamientos de las enfermedades bucales ya establecidas (23).

En la segunda mitad del siglo XX, y apoyada en la bonanza del "milagro económico mexicano", a la práctica dental se le facilitó el acceso a la tecnología de punta y al intercambio científico. El ejercicio de la profesión para los poco más de 1,500 cirujanos dentistas titulados que existían en el país en ese entonces, prácticamente garantizaba el éxito socioeconómico. La demanda de trabajo superaba con mucho a la oferta, contribuyendo esta situación de saturación ocupacional a que el cirujano dentista se aislara cada vez más en el estrecho individualismo del trabajo de consultorio. Sólo un escaso número de profesionales se preocupaban por desempeñar la práctica dental en el contexto de los problemas de salud más apremiantes del país. El Instituto Mexicano del Seguro Social, que poco antes había iniciado sus labores, prestaba en sus unidades aplicativas, igual que lo hace actualmente, un servicio dental limitado; lo mismo sucedía en los centros de salud y hospitales de la Secretaría de Salubridad y Asistencia. Hasta esta época la odontología en México se había limitado casi exclusivamente a una práctica de índole esencialmente curativa y restauradora. Debido a esta situación, las políticas en salud bucal no eran un tema de importancia. Hacia la sexta década del siglo XX la profesión entra en el campo de la prevención y de la salud pública, que hasta ese momento no estaban desarrolladas. Fue en esta época que el primer mexicano, Félix Leycegui, realizó estudios en la Universidad de Michigan sobre Odontología Sanitaria, apoyado por la Fundación Kellog. Leycegui fue profesor fundador de la asignatura en la UNAM y colaboró como asesor con la Secretaría de Salubridad y Asistencia, tanto a nivel central como estatal (23).

Al mismo tiempo la planta de universidades cambió radicalmente el panorama de los recursos humanos en odontología en el país. A partir de 1960 la profesión ve el surgimiento de nuevas escuelas dentales que se establecieron, en algunos casos más de una, en casi todos los estados de la República. Otro hecho destacado en la enseñanza odontológica es la organización de las especialidades. La demanda de inscripciones a la carrera de Odontología en la década 1970-80 se incrementa en forma exagerada, en parte por el espejismo del éxito profesional casi asegurado a corto plazo. Ante el temor de que esta situación se reflejara en una disminución de la calidad de enseñanza, en el centro del país se creó la especialización en docencia odontológica. Esto fue seguido por la firma de un convenio entre la UNAM y la Universidad de Texas que dio lugar a la maestría en odontología. A través de becas diversos estudiantes acuden a universidades en el extranjero a obtener los grados en diversas áreas de

\section{Revista Biomédica}




\section{Políticas de salud bucal en México}

la odontología. En la UNAM en 1975 se establece el primer curso de doctorado en odontología. Nuevas escuelas continuaron estableciéndose, tanto públicas como privadas; algunas, como la Universidad Autónoma Metropolitana y el Instituto Politécnico Nacional, ofrecen un enfoque diferente en la enseñanza de la odontología al incorporar a sus planes de studio y esfera de prioridades el servicio de la comunidad (23). Desde hace dos o tres décadas las escuelas y facultades de odontología han sido un importante auxiliar de los servicios de salud, aunque muchas veces no son tomadas en cuenta dentro de las estadísticas de los Sistemas de Salud, ya que en sus clínicas prestan atención curativa y preventiva a una proporción importante de la población del territorio nacional, principalmente urbana y de escasos recursos. La cuantificación de este impacto, la cantidad exacta de usuarios, y los resultados tangibles en servicios clínicos nunca han sido determinados de forma objetiva.

\section{Marco teórico referencial.}

La posesión de los bienes valorados en cualquier sistema social genera tanto alianzas como conflictos entre sus miembros y entre grupos sociales. Tales conflictos tienen origen en la relativa escasez de dichos bienes para satisfacer las necesidades sociales. La interacción de los individuos y las normas que rigen el comportamiento interno de los grupos no son suficientes para resolver todos esos conflictos. Cuando los problemas afectan de alguna manera a toda la sociedad y/o requieren decisiones que deben ser acatadas por todos sus miembros, las decisiones tienen que ser respaldadas por una autoridad que las haga obligatorias para toda la sociedad; estas son decisiones políticas (25). Antes de describir la evolución de la política de salud bucal en México, hagamos unas breves definiciones sobre los siguientes términos.

Política: es una actividad de decisión que de manera vinculante involucra la búsqueda del bienestar colectivo. Cuando hablamos de política nos referimos a aquella actividad específica que se relaciona con la adquisición, la organización, la distribución y el ejercicio del poder $(26,27)$.

Políticas públicas: es la política en algún nivel de gobierno que delinea el rango de posibilidades para las interacciones sociales. Algunos niveles pueden tener un precedente formal o legal sobre otros. Las políticas pueden ser puestas por el gobierno, las legislaturas, y las agencias reguladoras autorizadas por otras autoridades constituidas. Las instituciones de políticas supranacionales, como la Organización Mundial de la Salud, pueden dominar las políticas (27).

Política social: es aquella parte de la política general que tiene como meta específica promover el bienestar económico-social de la población. Incluye el conjunto de intervenciones públicas dirigidas a asegurar a los ciudadanos un estándar mínimo de salario, alimentación, salud, vivienda e instrucción, como derecho social y no como caridad. Se concibe también como un conjunto de arreglos, modelos y mecanismos orientados a la distribución de los recursos de acuerdo con algún criterio de necesidad (28).

Políticas publicas saludables: son las que mejoran las condiciones bajo las cuales viven las personas: sustento seguro, fijo, adecuado y sostenible, estilos de vida y ambientes, incluyendo hogar, educación, nutrición, intercambio de información, cuidado de niños, transporte, y servicios comunitarios necesarios, personal social y servicios de salud. Lo apropiado de la política puede ser medido por su impacto en la salud de población (27).

Con base en estas definiciones, podemos decir que las políticas de salud son el marco donde los individuos, las organizaciones, las empresas y las asociaciones de una comunidad pueden ejercer su actividad, además de que condicionan la forma y el contexto en el que se desenvuelven nuestras vidas. Las políticas tienen que ver con los procesos y el poder para situar los valores (recursos e ideas) en la sociedad. Las políticas se dan a varios niveles, 


\section{CE Medina-Solis, G Maupomé, L Avila-Burgos, R Pérez-Núñez, B Pelcastre-Villafuerte, y col.}

desde el que se produce a nivel legislativo como las decisiones que se toman internamente en las instituciones, centros de salud, etc.; se vuelven operacionales cuando en un grupo social decidimos realizar unas cosas y no otras, y cuando éstas las realizamos de un modo y no de otro.

\section{Epidemiología de la salud bucal: algunos resultados en México.}

Las enfermedades bucales constituyen un problema general de salud pública. La caries dental y las periodontopatías, por su magnitud y trascendencia, representan los principales problemas de salud bucal. La caries dental involucra un desequilibrio de las interacciones moleculares normales entre la superficie/subsuperficie del diente y el biofilm microbiano adyacente. Este desequilibrio se manifiesta en un cierto plazo como desmineralización acumulativa del diente que, si no se revierte, tiene el potencial de producir una cavitación en el esmalte y consecuentemente un daño colateral a la dentina y a la pulpa, culminando con la destrucción localizada de los tejidos duros del diente (29). La OMS la define como un proceso patológico de origen externo que se inicia después de la erupción y determina un reblandecimiento del tejido duro del diente, evolucionando hacia la formación de una cavidad. Las periodontopatías, por su parte, se caracterizan por producir inflamación y destrucción de los tejidos de soporte de los dientes. La etapa final de las periodontopatías ocasiona movilidad y pérdida de los dientes afectados (30-31).

En México solamente contamos con información limitada sobre la magnitud de los principales problemas bucales (32, 33). Esfuerzos aislados han permitido esbozar los rasgos generales de los problemas más importantes. En 1978, de La Rosa (34) realizó un estudio en niños de 6 a 15 años de edad en el que observó altos índices de caries, con promedios de 3.78 a 6.05 dientes afectados por caries a los 12 años de edad, según nivel socioeconómico. En una encuesta realizada por la Secretaría de Salud en 1980 en el DF se pudo observar que 95.5\% de los niños entre 6 a 14 años de edad padecía de caries dental. Jensen y Hermosillo (35) reportaron una prevalencia de caries en niños de 12 años casi de 100\% (97.3\%). Sánchez (36) reportó en niños de 12 años un promedio de casi 8 dientes afectados por caries y una prevalencia de 100\%. Entre 1987-1989, la Secretaría de Salud realizó el levantamiento de índices de caries en diversos Estados de la Republica, integrando la línea basal de caries para el Programa de Fluoración de la Sal de Mesa, observando que el índice de caries fue mayor a 3 en la mayoría de los estados (37). Maupomé (38) y colaboradores encontraron una alta prevalencia de caries en zonas rurales y periurbanas marginadas de varios estados de México. Irigoyen (39) en 1988 observó una prevalencia de caries de $90 \%$ en niños de 6 a 12 años de edad, con un promedio de 4.4 dientes afectados a los 12 años. Irigoyen (40) y colaboradores en 1984 y 1992, observaron índices de caries de 5.6 y 4.8, respectivamente, en dentición temporal de niños de 6 y 7 años de edad. Irigoyen y Szpunar mostraron que la prevalencia de caries se encontraba alrededor del 90\% en niños de 12 años (41). Diversos estudios epidemiológicos realizados recientemente indican que la prevalencia de caries en niños de México se encuentra entre 70\% y 85\% en la dentición permanente a los 12 años, y de $50 \%$ en la dentición temporal de los niños de 6 años, dependiendo de la población de que se trate (42-60). Según resultados parciales de la Primera Encuesta Nacional de Caries y Fluorosis Dental 1996-2000, la prevalencia de caries en niños de 6 años es $61.8 \%$ y el promedio de CPOD a los doce años es 2.23. En ambos casos se observa un alto porcentaje de lesiones cariosas no tratadas (33).

Con relación a las enfermedades periodontales, el conocimiento existente en México es aun más limitado. Sin embargo, diversos estudios las sitúan como una enfermedad de alta prevalencia, y que si bien se puede observar en sus etapas iniciales en los niños, se encuentran concentradas sobre todo en la población de adolescentes y adultos (60-69). Además, tanto la caries como las enfermedades

\section{Revista Biomédica}




\section{Políticas de salud bucal en México}

periodontales presentan un carácter acumulativo que se incrementa con la edad, hecho que justifica la importancia de prevenir estas enfermedades y evitar sus consecuencias (70).

\section{La salud bucal como elemento de la salud general.}

Desde hace ya varias décadas, la Organización Mundial de la Salud desarrolló una definición de salud como el "estado de completo bienestar físico, mental y social y no sólo la ausencia de enfermedad”. En años más recientes ha existido un considerable progreso en el desarrollo de medidas funcionales de salud, y la salud bucal forma parte de la salud integral, por lo que según la definición antes descrita, si se padece una enfermedad bucal se carece de salud general.

La salud bucal incluye el componente “dental” pero se relaciona con todo el complejo estomatognático. En otras palabras, la salud bucal no puede visualizarse únicamente como un problema dentario (71). Diversos estudios han cuantificado las consecuencias sociales de la enfermedad a través de las limitaciones en la vida diaria y la calidad de vida (72-74), aunque desafortunadamente la investigación en el estado de salud ha incluido primordialmente el espectro de condiciones severas y crónicas, y relativamente poca atención se le ha dado al impacto social de los problemas bucales. Es decir, la salud bucal ha sido medida tradicionalmente con base en la patología de los tejidos, con limitado reconocimiento de las implicaciones de salud, económicas y sociales de esas patologías $(72,75)$.

La percepción que explica por qué la incapacidad funcional causada por la enfermedad bucal no ha sido incorporada dentro de los indicadores del estado de salud general, es que las condiciones bucales son comúnmente experimentadas por la mayoría de los individuos, y como no amenazan la vida en comparación con otras condiciones más serias, los individuos no asumen el "rol de enfermo" para la mayoría de las condiciones dentales. Esto hace que la utilización de servicios de salud dental se vea disminuida y en muchos casos no sea importante dentro de la agenda de salud. Sin embargo, la evidencia sugiere que las condiciones dentales tienen un impacto significativo sobre la funcionalidad. Por ejemplo, datos de la Encuesta Nacional de Salud en Estados Unidos de Norteamérica indican que sólo los dolores dentales causan aproximadamente 17.7 millones de días de actividad restringida, lo que representa un serio problema de salud para la sociedad y un gasto importante para los sistemas de salud (72). Este impacto rebasa el ámbito puramente bucodental: varias investigaciones han demostrado la asociación existente entre las enfermedades bucales y las enfermedades sistémicas como endocarditis (76), enfermedad cerebrovascular (77-79), enfermedad coronaria (80, 81), infarto (82), hipertensión (83), diabetes mellitus (78), enfermedad respiratoria (78), y osteoporosis (78), así como bajo peso al nacer y nacimientos prematuros $(78,84)$, deficiencias nutricionales en los adultos (85), y cancer (8689).

\section{La política de salud bucal mundial.}

La salud en la población, aunque tiene su asiento biológico en cada individuo, tiene determinantes sociales y ambientales; por lo tanto, el mejoramiento de la salud puede consumarse a través de acciones deliberadas a nivel individual, de la prestación de servicios individuales y comunitarios, y de las intervenciones en salud pública. Algunos servicios públicos o privados, aunque no persiguen específicamente mejorar la salud, inciden en forma positiva o negativa sobre ella, y aunque su objetivo específico no es procurar cierto estado de salud, pueden mejorarla (90). La discusión de los factores incorporados en la primera parte de esta serie se abocará, sin embargo, a contrastar los factores de distintos niveles y características que están específicamente dirigidos a mejorar la salud de la población. Esto es, nos limitaremos a evaluar el impacto de los distintos niveles de políticas de salud, eventualmente por 


\section{CE Medina-Solis, G Maupomé, L Avila-Burgos, R Pérez-Núñez, B Pelcastre-Villafuerte, y col.}

separado, pero usualmente en conjunto.

En la reunión convocada por la Organización Mundial de la Salud en Alma Ata en 1978 se analizó el problema de la salud en el mundo y se adoptaron una serie de resoluciones en una declaración que lleva el nombre de esa conferencia. Estas resoluciones postularon la meta de "Salud para todos en el año 2000”, señalando que la posibilidad de lograrla dependía de la puesta en marcha de diferentes estrategias (91).

Después de la ratificación y adopción de la resolución de la Asamblea Mundial de la Salud en 1978, en 1981 la OMS adoptó como primer indicador global del estado de salud bucal un promedio de no más de 3 dientes cariados, perdidos u obturados a la edad de 12 años para el año 2000. Las siguientes metas fueron propuestas para el año 2000 por la OMS y la Federación Dental Internacional (FDI) en 1981: 50\% de los niños de 5-6 años debían estar libres de caries, 85\% de la población debía tener todos sus dientes en boca a los 18 años de edad, reducción en un $50 \%$ de los niveles de personas edéntulas a los 35-44 años de edad, y la reducción en un $25 \%$ de las personas edéntulas a los 65 años de edad (92). En 1983 la salud bucal fue declarada como parte de la "Estrategia Salud para Todos" (resolución WHA36.14) y en 1989 la OMS aprobó la promoción de la salud bucal como parte integrante de "Salud para todos para el año 2000" (WHA42.39). Además, el Día Mundial de la Salud en 1994 fue dedicado a la salud bucal, lo que refleja la importancia conectada a este tema (93).

A pesar de estas prioridades y metas, las condiciones de salud bucal en el contexto latinoamericano se expresan como un verdadero mosaico epidemiológico. En este mosaico se combinan problemas característicos de las llamadas culturas subdesarrolladas con las de avanzado desarrollo, en las cuales la estructura socioeconómica y situación geográfica acusan marcadas contradicciones, entre otros aspectos, por las condiciones de salud de la población y, como en el caso de la salud bucal, por las posibilidades de acceso a los servicios de salud bucal (71). Como resultado de las desigualdades en salud entre los grupos sociales, en América Latina como conjunto no se lograron las metas en salud bucal propuestas para el año 2000 por la OMS/FDI. En México, según la "Primera Encuesta Nacional de Caries y Fluorosis", 3 de los 21 estados con datos disponibles en el 2000 no cumplieron con dicha meta (14.3\%) (94).

En el año 2000 como resultado de la distribución de la caries dental en la población (la cual presenta un sesgo o una concentración de la enfermedad en un segmento de la población en alto riesgo) se propuso un nuevo índice de caries y nuevas metas. El índice propuesto es el Índice de Caries Significante ( $\mathrm{SiC}$ ), que pone mayor atención en el tercio de la población con la mayor experiencia de caries. La meta propuesta para el 2015 es que a los 12 años de edad el SiC no sea mayor de 3 (95). Asimismo, la FDI, la OMS y la Asociación Internacional para la Investigación Dental (IADR) han presentado nuevas metas para el año 2020. Este documento contiene propuestas para nuevas metas globales de salud bucal, objetivos y población a las que se dirige. Es un marco de referencia útil para los planificadores de salud regionales, nacionales y locales, al mismo tiempo que no pretende ser prescriptivo (96). En la actualidad, la política de salud bucal mundial es la de continuar con la mejora de la salud bucal en el siglo XXI (93).

\section{La política de salud bucal en México.}

La SSA creó la Dirección de Odontología en la década de los 60 con los objetivos principales de implantar procedimientos preventivos en los diferentes departamentos dentales de los Centros de Salud del país; difundir, especialmente a nivel escolar, los principios higiénicos para fomentar la salud dental; y estudiar la viabilidad de la fluoración del agua potable o de la sal de consumo. Durante este tiempo se fomentó la actualización de todo el personal odontológico de las instituciones que formaban el Sistema de Salud. Se efectuaron también análisis químicos para determinar el

\section{Revista Biomédica}




\section{Políticas de salud bucal en México}

contenido del ión flúor en el agua de diferentes ciudades del país, y se inició la fluoruración del agua en las plantas potabilizadoras de Los Mochis, Sin., Veracruz, Ver., y el conjunto urbano NonoalcoTlatelolco, en el DF. Desafortunadamente, los cambios administrativos y financieros provocaron la desaparición de esas primeras y únicas plantas fluoruradoras antes de que pudieran evaluarse sus logros.

Al presentar la población infantil altos índices de caries y ante la imposibilidad percibida de brindar atención bucal integral a toda la población durante la década de los 70 y 80, debido a los altos costos, se consideró conveniente instaurar programas preventivos de amplia cobertura para la población general. Debido a que en el país no toda la población tiene servicio de agua intradomiciliaria, la fluoruración de la sal doméstica representaba un mejor vehículo de distribución. En 1988 se normó y reglamentó la fluoruración de la sal $(97,98)$. En marzo de 1981 se decretó en México la fluoración de la sal (99). Para la operación del Programa Nacional de Fluoruración de la Sal se estableció un acuerdo entre la SSA, la Secretaria de Comercio y Fomento Industrial y la Asociación Mexicana de la Industria Salinera, para la producción, distribución y comercialización de la sal en los estados donde el abastecimiento de agua potable contiene flúor en cantidad menor a la óptima (0.7-1.59 ppm) (100). Este programa estipuló cantidades recomendadas por organismos internacionales tras la experiencia de diversos países en la fluoruración de la sal para tener efectos carioprofilácticos y cariostáticos deseables (101).

Los programas de fluoruración a nivel sistémico y de salud pública no fueron la única estrategia preventiva en funciones. En 1983 la preocupación por la salud en escolares condujo a la realización del Primer Congreso Higiénico Pedagógico. De entonces a la actualidad diversas estrategias, acciones y modelos se han propuesto para proteger al niño en esa etapa de la vida (102). Actualmente las acciones preventivas y educativas dirigidas a la niñez escolar mexicana se basan en la norma "NOM-009-SSA2-1993 para el fomento de la salud del escolar", en la cual se puede observar un componente sobre salud bucal (103). Con relación a la política de salud bucal dentro de las políticas nacionales de salud, es hasta 1988 que apareció por primera vez el componente de salud bucal en el informe de labores de la Secretaría de Salud como una parte de la recientemente creada Dirección de Fomento a la Salud (104).

Así, entre lo más destacado de la política de salud bucal en la década de los 80 podemos mencionar: 1) en 1981, la publicación del Reglamento de Yodación y Fluoruración de la Sal, en el Diario Oficial de la Federación en México; 2) en 1984, la definición de la fluoruración de la sal en el Programa Nacional de Salud; 3) en 1985, la promoción legal y económica para la fluoruración de la sal, por instancia del Instituto de Salud en el Estado de México; 4) en 1987, la creación del Comité Interinstitucional para la Fluoruración y elaboración del Programa Nacional en México; 5) la segunda publicación y reformas al reglamento para la sal yodada y fluorurada; 6) la publicación de la Norma Oficial Mexicana NOM-F-8-1988; y 7) la puesta en marcha de modelos de salud en escolares y del sub-programa de salud bucal (105). En 1990 la Dirección General de Fomento a la Salud puso en marcha el modelo de salud bucal para escolares en las principales ciudades del país, buscando involucrar de manera integral los aspectos educativo, preventivo, curativo y de participación social, para mejorar la salud bucal de la población escolar. Igualmente, con este modelo se pretendió fomentar en la comunidad escolar la adquisición de conocimientos y el desarrollo de conceptos, conductas, actitudes y hábitos positivos relacionados con la salud bucal (31).

Entre los doce programas prioritarios del Programa Nacional de Promoción y Cuidado de la Salud entre 1989-1994, existían dos relacionados con la salud bucal. Estos eran el programa de salud escolar y el programa de fluoruración de la sal. En esta época se puso en marcha el Programa Nacional Educativo Preventivo contra Caries y

Vol. 17/No. 4/Octubre-Diciembre, 2006 


\section{CE Medina-Solis, G Maupomé, L Avila-Burgos, R Pérez-Núñez, B Pelcastre-Villafuerte, y col.}

Parodontopatías en Preescolares y Escolares para mejorar la salud bucal de la población escolar (106). Asimismo, en la década de los 90 se publica la NOM-013-SSA2-1994, para la prevención y control de las enfermedades bucales. Esta norma tiene por objetivo establecer los métodos, técnicas y criterios de operación del Sistema Nacional de Salud, con base en los principios de la prevención de la salud bucal a través de la operación de las acciones para fomento de la salud, la protección específica, el tratamiento, la rehabilitación y el control de las enfermedades bucales de mayor frecuencia $(107,108)$. En 1995 se publicó la NOM-040-SSA1-1993, Bienes y servicios, sal yodada y sal yodada fluorurada, la cual establece las especificaciones sanitarias de la sal para consumo humano y animal (109).

En 1996 se iniciaron las Semanas Nacionales de Salud Bucal, programándose dos para cada año. En estas actividades se unen los esfuerzos de instituciones del Sector Salud, además de asociaciones gremiales y la Secretaría de Educación Pública. El objetivo fue intensificar todas las actividades preventivas dentro de un método unificado y normado, enfocándose a la caries dental, la gingivitis, las maloclusiones, el cáncer bucal y los padecimientos dentofaciales que restan calidad de vida a los individuos. Esta estrategia se realiza periódicamente en los 32 estados, dentro de las unidades clínicas y en escuelas de educación primaria. Las características del programa incluyen respetar las políticas internas de cada organismo participante; que cada estado e institución organice, según sus necesidades, un curso de capacitación previo para todo el personal participante en la realización de las acciones específicas de la "Semana Nacional de Salud Bucal"; que la unificación de criterios sea únicamente para lograr las metas planteadas; que cada organismo decida sobre el personal y recursos que pueda destinar para el proyecto operativo; y que las metas sean calculadas de acuerdo a los insumos y recursos de los participantes.

En abril de 1998 el Programa de Salud Bucal fue incluido como programa prioritario en las políticas nacionales de salud (102).

La política de salud bucal en el periodo 20002006 reconoce que en México las principales enfermedades bucales se encuentran todavía con una alta incidencia y prevalencia, al igual que como enfermedades del rezago que afectan principalmente a los pobres. Entre ellas destacan la caries dental y la enfermedad periodontal, que según el Plan Nacional de Salud (PNS) afectan a 90 y 70 por ciento de la población, respectivamente. Para su disminución y atención se han diseñado las siguientes actividades dentro del Programa Nacional de Salud (PNS) 2001-2006 (33, 94), desde donde se dictan las principales políticas de salud en México y en correspondencia con la política social del Plan Nacional de Desarrollo (110): promoción de la salud bucal de la población; fortalecimiento de los programas institucionales a nivel nacional; intensificación de las actividades de salud bucal durante las dos Semanas Nacionales de Salud Bucal; promoción del desarrollo de investigaciones en salud bucal; ampliación de la cobertura del servicio estomatológico a zonas marginadas de difícil acceso aplicando el Tratamiento Restaurativo Atraumático; fortalecimiento de la coordinación entre las áreas relacionadas con el Programa de Fluoruración de la Sal de Mesa. Con estas actividades se espera ampliar la cobertura a la población y alcanzar las metas de alto impacto, que son disminuir la prevalencia de caries a los seis años de edad al 50 por ciento, y obtener a los 12 años de edad un índice de dientes permanentes cariados, perdidos y obturados (CPOD) de tres.

Dentro de la Política Nacional de Salud se generó el Programa de Acción Salud Bucal, que en su objetivo general pretende mejorar la salud bucal de la población mexicana, contribuyendo al abatimiento de las enfermedades bucales de mayor incidencia y prevalencia. La misión de este programa de acción es la de disminuir las enfermedades bucales de mayor prevalencia e incidencia a través de establecer, fortalecer

\section{Revista Biomédica}




\section{Políticas de salud bucal en México}

y vigilar políticas, estrategias y programas, así como evaluar las condiciones de la salud bucal para mejorar y ampliar equitativamente la atención a la población con ética, calidad y eficiencia (94). La mejora de la salud bucal en la población se pretende conseguir a través de varios componentes; estos son: el componente de salud bucal del preescolar y escolar, de fluoruración de la sal de mesa, el aspecto curativo-asistencial y la normatividad. Estos elementos presentan a su vez objetivos generales y específicos, estrategias, metas, acciones estratégicas y acciones a mediano plazo, para cumplir con lo marcado en la política nacional de salud bucal. Es de suponerse que, dada esta estructuración, será factible evaluar el cumplimiento de metas, objetivos y programas, lo cual no ha sido cabalmente realizado con otros programas en el pasado.

En 2003 se puso en marcha el Sistema Nacional de Cartillas de Salud que aparentemente es la política de salud más reciente. En estas cartillas los individuos podrán llevar un seguimiento personalizado y continuo de las acciones de prevención para la salud que reciban en cada etapa de la vida. Este sistema está compuesto de cuatro elementos: 1) la cartilla nacional de vacunación (que ya estaba siendo empleada desde 1978), 2) la cartilla de salud de la mujer, 3) la cartilla nacional del hombre, y 4) la cartilla nacional del adulto mayor. En las últimas tres uno de los rubros básicos es la salud bucal (111).

\section{Discusión y conclusiones.}

Es indudable que en las últimas décadas algunos sectores de la población de México han presentado una mejoría en su estado de salud bucal. Esta mejora no hubiese sido posible sin la promulgación de políticas de salud bucal, con las estrategias y programas que de ella se desprenden. Este esfuerzo ha coincidido en lo general con las propuestas de organismos internacionales como la Organización Mundial de la Salud y la Organización Panamericana de la Salud. No obstante, es evidente que aún queda mucho por hacer en términos de asegurar un buen estado de salud bucodental, apropiado a las expectativas y necesidades clínicas de los distintos grupos poblacionales. Asimismo, algunos de estos logros han estado dirigidos sólo a una parte de la salud bucal, y no a todas las enfermedades y condiciones a las que hace referencia la política de salud bucal. Por otra parte, en algunas ocasiones los diferentes programas de salud bucal no han sido bien planificados o realizados, o han estado básicamente enfocados a poblaciones más aparentes para los planificadores de servicios.

Algunas de las áreas precisan de atención especial. Entre ellas destaca el uso racional y cuidadoso de los fluoruros sistémicos. Cuando se usan en forma adecuada, los fluoruros son un agente seguro para la prevención y el control de la caries dental. Cuando se dispone de múltiples fuentes de fluoruro (como es común en el mundo actual), es posible que una minoría de la población ingiera una mayor cantidad a la mínima segura durante periodos de susceptibilidad en los que los dientes se mineralizan. Esto plantea un riesgo para la población abierta, dando lugar a fluorosis dental (112). El fluoruro en las últimas décadas ha sido un factor clave para la disminución de la caries dental que se ha venido observando en diversos países del mundo, incluyendo a México. Sin embargo, la disponibilidad de fluoruros a través de diversas fuentes como las aplicaciones profesionales, programas masivos y continuos de salud bucal, alimentos, bebidas y pastas dentales, entre otras, se ha ido incrementando. Así, hoy en día virtualmente todos los niños mexicanos se encuentran expuestos a diversas fuentes de fluoruro. En los países industrializados la disminución de la caries dental ha ido acompañada por un incremento en la prevalencia de fluorosis dental leve o muy leve (que es la primera señal de una intoxicación crónica por fluoruro), la cual es la principal forma de presentación en México (113126). Niveles más severos pueden tener un efecto cosmético negativo en los individuos, y pueden afectar las relaciones sociales y tener repercusiones 
CE Medina-Solis, G Maupomé, L Avila-Burgos, R Pérez-Núñez, B Pelcastre-Villafuerte, y col.

psicológicas. La disponibilidad de los fluoruros en los programas de salud bucal debe estar en relación directa con las necesidades preventivas de la población bajo sistemas de vigilancia continua, considerando un enfoque de riesgo a caries y una vigilancia continua de la relación entre efectos deseables e indeseables de esta medida de salud pública. A pesar de la importancia que reviste esta evaluación constante y la considerable cantidad de publicaciones de envergadura menor en la literatura dental mexicana, no existe un acervo de información que permita establecer objetivamente las características epidemiológicas de la fluorosis dental en México. Considerando que la población mexicana que vive a más de $2000 \mathrm{~m}$ sobre el nivel del mar está en un riesgo particularmente alto de sufrir fluorosis dental (121), esta es una omisión grave en los programas de vigilancia epidemiológica.

Las políticas de los organismos internacionales que velan por la salud de la población han generado un notable incremento de las expectativas de vida. Por tanto, la proporción de personas de la tercera edad aumenta progresivamente y las previsiones apuntan hacia la continuidad de esta tendencia. Entre los retos a mediano y corto plazo está el determinar las necesidades de atención y el estado de salud bucal de población mexicana de la tercera edad, de la cual no es bien conocido el estado de salud. Debido al aumento en el número de personas de la tercera edad México requerirá en el futuro un adecuado plan para hacer frente a las necesidades que presenta este grupo poblacional.

Otro aspecto importante es la educación profesional y la formación de recursos humanos en esta área de la salud, la cual no ha sido tomada en cuenta dentro de la política de salud bucal. Podemos afirmar que se observa un desencuentro en lo que se enseña en las universidades y lo que requiere la población para mejorar la salud buco-dental. Se ha mencionado que entre los retos en materia de educación en la preparación de las nuevas generaciones de odontólogos estarían ciertos aspectos clave $(127,128)$, entre ellos: 1$)$ realizar una mejor planeación y normar adecuadamente la educación dental para la formación de recursos humanos, de acuerdo con las necesidades de la población (de distintas clases sociales, y en medios urbanos y rurales); 2) instituir la prevención primaria y las medidas de promoción de la salud como prioridades en la educación universitaria y en la práctica profesional, con la finalidad de equilibrar los enfoques de rehabilitación actuales; y 3) modificar el énfasis actual en la formación de recursos humanos, dando prioridad a la creación de profesionistas comunitarios. A mediano plazo, estos nuevos esquemas educacionales proveerían de un modelo de atención bucodental más acorde con las necesidades clínicas y con la realidad socioeconómica de la población que el modelo actual. Los esquemas educacionales actuales repercuten en el ejercicio de la profesión, y tal vez ha llegado el momento de estudiar fórmulas que permitan reconvertir los recursos humanos disponibles que se graduaron en los últimos 25 años, y que no se han actualizado ni han estado particularmente activos en la vida profesional (127-131).

El mercado profesional odontológico ha estado gobernado por la oferta exagerada, y la demanda insatisfecha en algunos puntos. Esta es una buena razón para apoyar la introducción de cambios en las políticas de salud bucodental en el país. Con estos cambios podríamos reducir las brechas de salud bucal que se encuentran entre los sectores urbano y rural, así como las diferencias en la distribución de la enfermedad bucal, la utilización y la cobertura de este tipo de servicios entre las clases sociales (132-135). Por todo lo anterior podemos decir que, grosso modo, los esquemas de educación dental en las universidades, los servicios clínicos en el sector público y privado, y los esquemas preventivos de cubrimiento masivo conforman un mosaico inconexo.

A partir de este trabajo de revisión es posible vislumbrar el siguiente paso que sería una evaluación de la política de salud bucal de México

\section{Revista Biomédica}




\section{Políticas de salud bucal en México}

utilizando una metodología rigurosa, incluyendo variables de diversos tipos. Si bien se ha observado una mejoría en la salud bucal de algunos segmentos de la población, esto no ha sido cuantificable debido a la falta en la disponibilidad de datos que permitan una estimación correcta. Además, hay que tener en cuenta que los índices utilizados a lo largo del tiempo han sufrido cambios, oscureciendo así el panorama de los logros de la salud bucodental. Es preciso determinar en qué sectores de la población ha sido mayor el impacto de la política.

\section{REFERENCIAS.}

1. Secretaría de Salud. Subsecretaría de Prevención y control de enfermedades. Programa de Salud Bucal. SSA México; 2000.

2. Anderson RJ. The reduction of dental caries prevalence in English schoolchildren. J Dent Res 1982; 61(Special Iss): 1311-6.

3. Downer MC. Secular changes in caries dental experience in Scotland. J Dent Res 1982; 61(Special Iss):1336-9.

4. Holst A, Braune K, Kjellberg M. Changes in caries experience among 6-year-olds in Blekinge, Sweden between 1994 and 2000. Swed Dent J 2004; 28:129-35.

5. Pieper K, Schulte AG. The decline in dental caries among 12-year-old children in Germany between 1994 and 2000. Community Dent Health 2004; 21:199-206.

6. Carvalho JC, D’Hoore W, Van Nieuwenhuysen JP. Caries decline in the primary dentition of Belgian children over 15 years. Community Dent Oral Epidemiol 2004; 32:277-82.

7. Marthaler TM. Changes in dental caries 1953-2003. Caries Res 2004; 38:173-81.

8. Birkeland JM, Haugejorden O, von der Fehr FR. Analyses of the caries decline and incidence among Norwegian adolescents 1985-2000. Acta Odontol Scand 2002; 60: 281-9.

9. Beltran-Aguilar ED, Estupiñan-Day S, Baez R. Analysis of prevalence and trends of dental caries in the Americas between the 1970s and 1990s. Int Dent J 1999; 49:322-9.

10. Bonecker M, Cleaton-Jones P. Trends in dental caries in Latin American and Caribbean 5-6- and 11-13-year- old children: a systematic review. Community Dent Oral Epidemiol 2003; 31:152-7.

11. Manji F, Fejerscov O. Dental caries in developing countries in relation to the appropriate use of fluoride. J Dent Res 1990; 69:733-41.

12. Nadanovsky $P$, Sheiham A. Relative contribution of dental services to the changes in caries levels of 12-year-old children in 18 industrialized countries in 1970's and early 1980`s. Community Dent Oral Epidemiol 1995; 23:331-9.

13. Brunelle A, Carlos JP. Changes in the prevalence of dental caries in US schoolchildren, 1961-1980. J Dent Res 1982; 61(Special Iss):1346-51.

14. Newbrum E. Effectiveness of water fluoridation. J Public Health Dent 1989; 49:279-89.

15. Maupomé G, Clark DC, Levy SM, Berkowitz J. Patterns of dental caries following the cessation of water fluoridation. Community Dent Oral Epidemiol 2001; 29:37-47.

16. Cypriano S, Pecharki GD, de Souza ML, Seichi.Wada R. A saúde bucal de escolares residentas em locais com ou sem fluoretacao nas águas de abastecimento público na regiao de Sorocaba, Sao Paulo, Brasil. Cad Saúde Pública 2003; 19:1063-71.

17. Ainamo J, Parviainen K. Influence of increased toothbrushing frequency health in low, optimal and high fluoride areas in Finland. Community Dent Oral Epidemiol 1989; 17:296-9.

18. Mayanagi H, Saito T, Kamiyama K. Cross-sectional comparisons of dental caries trends in nursery schoolchildren in Sendai, Japan. Community Dent Oral Epidemiol 1995; 23:344-9.

19. Lalonde M. A new perspective on the health of the Canadians: a working document. Ottawa: Government of Canada, 1974.

20. Van-Hersen LM, Reijneveld SA, Gunning-Schepers LJ. Rationalising chances of success in intersectoral health policy making. J Epidemiol Community Health 2001; 55: 342-7.

21. Zimbrón-Levy A, Feingold-Steiner M, Sanfilippo J. Apuntes sobre la práctica de la odontología en el Mexico Colonial (1530-1800). Centro Regional de Investigaciones Multidisciplinarias - UNAM. Cuernavaca, México; 1989. 


\section{CE Medina-Solis, G Maupomé, L Avila-Burgos, R Pérez-Núñez, B Pelcastre-Villafuerte, y col.}

22. Zimbrón-Levy A, Feingold-Steiner M. Odontología contemporánea en México: etapa inicial (1900-1930). Centro Regional de Investigaciones Multidisciplinarias - UNAM. Cuernavaca, México; 1989.

23. Zimbrón-Levy A, Feingold-Steiner M. Breve historia de la odontología en México. Centro Regional de Investigaciones Multidisciplinarias - UNAM. Cuernavaca, México; 1990.

24. Guerrero-Santoyo MR. El Boletín Odontológico Mexicano: Una propuesta para el estudio de la historia de la odontología en México. Rev ADM 2003; 60:155-9.

25. Organización Panamericana de la Salud. Centro Panamericano de Planificación de la Salud. Formulación de políticas de salud. Santiago de Chile, 1975.

26. Cisneros I. Política. En: Léxico de la política. FCE, México, 2000. pp 554-8.

27. Milio N. Glossary: healthy public policy. J Epidemiol Community Health 2001; 55:622-3.

28. Gordon S. Políticas públicas. En: Léxico de la política. FCE, México, 2000. pp 582-6.

29. Pitts NB, Stamm JW. International Consensus Workshop on Caries Clinical Trials (ICW-CCT) - Final consensus statements: Agreeing where the evidence leads. J Dent Res 2004; 83(Spec Iss C):C125-8.

30. Organización Mundial de la Salud. Metodología y programa de prevención de las enfermedades buco-dentales. Serie de Informes Técnicos Número 173. OMS; 1984.

31. Secretaría de Salud. Subsecretaría de Servicios de Salud. Dirección General de Fomento de la Salud. Modelo de Salud Bucal para Escolares. SSA México; 1990.

32. Moreno-Altamirano A. Cartas al editor. Propone aplicar un índice comunitario de fluorosis. Salud Publica Mex 2001; 43:179-81.

33. Secretaría de Salud. Programa Nacional de Salud 20012006. SSA México; 2001. p. 97.

34. De la Rosa M. Dental caries and socioeconomic status in Mexican children. J Den Res 1978; 57:453-7.

35. Jensen K, Hermosillo-Jensen GG. Salud Dental: problemas de caries dental, higiene bucal y gingivitis en la población marginada metropolitana de México. Bol Of Sanit Panam 1983; 94:587-603.

\section{Revista Biomédica}

36. Sánchez-Perez L. Caries dental en el sur del DF: Pract Odontol 1987; 8:25-30.

37. Secretaría de Salud. Programa Nacional de Salud 20012006. Estrategia: reducir los rezagos en salud que afectan a los pobres. Programa de Acción: Salud Bucal. SSA México; 2001.

38. Maupomé-Carvantes G, Borges-Yañez SA, LedesmaMontes C, Herrera-Echauti R, Leyva-Huerta ER, NavarroAlvarez A. Prevalencia de caries en zonas rurales y periurbanas marginadas. Salud Publica Mex 1993; 35:357-67.

39. Irigoyen-Camacho ME. Caries dental en escolares del Distrito Federal. Salud Publica Mex 1997; 39:133-6.

40. Irigoyen-Camacho ME, Molina Feichero N, Villanueva Arriaga R, García-López S. Cambios en los índices de caries dental en escolares de una zona de Xochimilco, México: 1984-1992. Salud Publica Mex 1995; 37:430-6.

41. Irigoyen ME, Szpunar SM. Dental caries status of 12year-old students in the state of Mexico. Community Dent Oral Epidemiol 1994; 22:311-4.

42. Dufoo S, Maupomé G, Diez-de-Bonilla J, Hernandez JC. Caries experience in a selected patient population in Mexico City. Community Dent Oral Epidemiol 1996; 24:298-9.

43. Sánchez-Flores I, Nava-Romero J. Niveles de infección de streptococcus mutans y caries dental en un grupo de niños de 12 años de edad. Pract Odontol 1996; 17:6-9.

44. Molina-Frechero N, Irigoyen ME. Streptococcus mutans y prevalencia de caries en una población escolar. Pract Odontol 1996; 17:19-24.

45. Tello-de-Hernández TJ, Hernández-Pereira J, GutiérrezGarcía N. Epidemiología oral de tejidos duros y blandos en escolares del Estado de Yucatán, México. Rev Biomed 1997; 8:65-79.

46. Osorio-Rosado G, Hernández-Pereira J. Prevalencia de caries dental en dos grupos escolares de 6 a 12 años de edad de Mérida y Cancún. Rev ADM 1998; 55:227-34.

47. Vallejos-Sánchez A, Pérez-Olivares S, Casanova-Rosado A, Gutiérrez-Salazar M. Prevalencia, severidad de fluorosis y caries dental en una población escolar de seis y 12 años de edad en la Ciudad de Campeche, 1997-98. Rev ADM 1998; 55:266-71. 


\section{Políticas de salud bucal en México}

48. Maupomé G. An introspective qualitative report on dietary patterns and elevated levels of dental decay in a deprived urban population in northern Mexico. ASDC J Dent Child 1998; 65:276-85.

49. Irigoyen ME, Maupomé G, Mejía AM. Caries experience and treatment needs in 6 to 12 year-old urban population in relation to socioeconomic status. Community Dent Health 1999; 16:245-9.

50. Mendoza-Roaf P, Pozos-Radillo E, Balcazar-Partida N, Valadez-Figueroa I, Pando-Moreno M, Guerra JF. Caries dental en escolares de seis y 12 años de edad y su relación con el nivel socioeconómico y sexo en Guadalajara. Pract Odontol 1999; 20:12-8.

51. Moreno-Altamirano A, Carreón-García J, Alvear-Galindo G, López-Moreno S, Vega-Franco L. Riesgo de caries en escolares de escuelas oficiales de la Ciudad de México. Rev Mex Pediatr 2001; 68:228-33.

52. Medina-Solís CE, Casanova-Rosado AJ, CasanovaRosado JF, Vallejos-Sánchez AA, Segovia-Villanueva AR, Estrella-Rodríguez RJ. Caries dental e indicadores de riesgo en niños de guarderías del Instituto Mexicano del Seguro Social, Campeche, México, en 1999. Bol Med Hosp Infant Mex 2002; 59:419-29.

53. Juárez-López ML, Hernández-Guerrero JC, JiménezFarfán D, Ledesma-Montes C. Prevalencia de fluorosis dental y caries en escolares de la ciudad de México. Gac Med Mex 2003; 139:221-5.

54. Herrera MS, Medina-Solís CE, Rosado-Vila G, MinayaSánchez M, Vallejos-Sánchez AA, Casanova-Rosado JF. Prevalencia, severidad de caries y necesidades de tratamiento en preescolares de una comunidad suburbana de Campeche2001. Bol Med Hosp Infant Mex 2003; 60:189-96.

55. Casanova-Rosado AJ, Medina-Solís CE, CasanovaRosado JF, Vallejos-Sánchez AA, Maupomé G, ÁvilaBurgos L. Dental caries and associated factors in Mexican schoolchildren aged 6-13 years. Acta Odontol Scand 2005; 63:245-51.

56. Segovia-Villanueva A, Estrella-Rodríguez R, MedinaSolís CE, Maupomé G. Severidad de caries en preescolares bajo un programa de odontología preventiva. Rev Salud Pública (Bogotá) 2005; 7:56-69.

57. Segovia-Villanueva A, Estrella-Rodríguez R, MedinaSolís CE, Maupomé G. Dental caries experience and factors among preschoolers in Southeastern Mexico. J Public Health
Dent 2006; 66: 88-91.

58. Beltrán-Valladares P, Cocom-Tum H, Casanova-Rosado JF, Vallejos-Sánchez AA, Medina-Solís CE, Maupomé G. Caries prevalence and some associated factors in 6-9-yearold schoolchildren in Campeche, Mexico. Rev Biomed 2006; 17:25-33.

59. Vallejos-Sánchez AA, Medina-Solís CE, CasanovaRosado JF, Maupomé G, Minaya- Sánchez M, Pérez-Olivares S. Caries increment in the permanent dentition of Mexican children in relation to prior caries experience on permanent and primary dentitions. J Dent 2006; 34:709-15.

60. Medina-Solís CE, Maupomé G, Pelcastre-Villafuerte B, Avila-Burgos L, Vallejos-Sánchez AA, Casanova-Rosado AJ. Desigualdades socioeconómicas en salud bucal: caries dental en niños de 6 a 12 años de edad. Rev Invest Clin 2006; 58: 296-304.

61. Gonzalez M, Cabrera R, Grossi SG, Franco F, Aguirre A. Prevalence of dental caries and gingivitis in a population of Mexican schoolchildren. Community Dent Oral Epidemiol 1993; 21:11-4.

62. De la Teja AE, García-Dehesa DM, López-Morteo VM, Gutiérrez-Castrellón P, Motoro-Soto M. Gingivitis en escolares de nivel socioeconómico pobre. Acta Pediatr Mex 1999; 20:280-3.

63. Irigoyen E, Velázquez C, Zepeda M, Mejía A. Caries dental y enfermedad periodontal en un grupo de personas de 60 o más años de edad de la Ciudad de México Rev ADM 1999; 56:64-9.

64. Castellanos-Suárez JL, Díaz-Guzmán LM. Periodontitis crónica y enfermedades sistémicas. Rev ADM 2002; 59: 121-7.

65. Orozco-Jaramillo RE, Peralta-Lailson H, Palma-Montoya GG, Pérez-Rodríguez E, Arroniz-Padilla S, LlamosasHernández E. Prevalencia de gingivitis en adolescentes en el municipio de Tlalnepantla. Rev ADM 2002; 59:16-21.

66. Murrieta-Pruneda JF, Juárez-López LA, Linares-Vieyra C, Zurita-Murillo V. Prevalencia de gingivitis en un grupo de escolares y su relación con el grado de higiene oral y el nivel de conocimiento sobre salud bucal demostrado por sus madres. Bol Med Hosp Infant Mex 2004; 61:44-54.

67. Juárez-López MLA, Munieta-Pruneda JF, TeodosioProcopio E. Prevalencia y factores de riesgo asociados a enfermedad periodontal en preescolares de la Ciudad de

Vol. 17/No. 4/Octubre-Diciembre, 2006 


\section{CE Medina-Solis, G Maupomé, L Avila-Burgos, R Pérez-Núñez, B Pelcastre-Villafuerte, y col.}

México. Gac Méd Méx 2005; 141:185-9.

68. Borges-Yanez SA, Irigoyen-Camacho ME, Maupome G. Risk factors and prevalence of periodontitis in communitydwelling elders in Mexico. J Clin Periodontol 2006; 33: 184-94.

69.- Borges-Yanez SA, Maupome G, Martinez-Gonzalez M, Cervantez-Turrubiante L, Gutierrez-69. Robledo LM. Dietary fiber intake and dental health status in urbanmarginal, and rural communities in central Mexico. J Nutr Health Aging 2004; 8:333-9.

70. Medina-Solís CE, Pérez-Núñez R, Maupomé G, Casanova-Rosado JF. Edentulism among Mexicans 35 years old and older, and associated factors. Am J Public Health 2006; 96:1578-81.

71. Organización Panamericana de la Salud. Organización Sanitaria Panamericana, Oficina Regional de la Organización Mundial de la Salud. Desarrollo y fortalecimiento de los sistemas locales de salud. La salud bucal. HSD/SILOS-22. OPS; 1993.

72. Reisine ST. Dental health and public Policy: The social impact of dental disease. Am J Public Health 1985; 75: 27-30.

73. Allen PF. Association between diet, social resources and oral health related quality of life in edentulous patients. J Oral Rehabil 2005; 32:623-8.

74. Astrom AN, Haugejorden O, Skaret E, Trovik TA, Klock KS. Oral impacts on daily performance in Norwegian adults: validity, reliability and prevalence estimates. Eur J Oral Sci 2005; 113:289-96.

75. Sheiham A. Oral health, general health and quality of life [editorial]. Bull World Health Organ 2005; 644-5.

76. Strom BL, Abrutym E, Berlin JA, Kinman JL, Feldman RS, Stolley PD, et al., Dental and cardiac risk factors for infective endocarditis. A population based, case control study. Ann Intern Med 1998; 129:761-9.

77. Joshipura KJ, Hung HC, Rimm EB, Willet WC, Ascheiro A. Periodontal disease, tooth loss, and incidence of ischemic stroke. Stroke 2003; 34:47-52.

78. Amar S, Han X. The impact of periodontal infection on systemic disease. Med Sci Monit 2003; 9:291-9.

79. Elter JR, Offenbacher S, Toole JF, Beck JD. Relationship of periodontal disease and edentulism to stroke/TIA. J Dent Res 2003; 82:998-1001.

80. Fong IW. Emerging relations between infectious diseases and coronary disease and atherosclerosis. CMAJ 2000; 163: 49-56.

81. Luis-Delgado O, Echeverría-García JJ, Berini-Aytés L, Gay-Escoda C. La periodontitis como factor de riesgo en los pacientes con cardiopatía isquémica. Med Oral 2004 (Esp); 9:125-37.

82. Renvert S, Ohlsson O, Persson S, Lang NP, Persson GR. Analysis of periodontal risk profiles in adults with or without a history of myocardial infarction. J Clin Periodontol 2004; 31:19-24.

83. Taguchi A, Sanada M, Suei Y, Ohtsuka M, Lee $\mathrm{K}$, Tanimoto $\mathrm{K}$, et al. Tooth loss is associated with an increased risk of hypertension in postmenopausal women. Hypertension 2004; 43:1297-300.

84. Mokeem SA, Molla GN, Al-Jewair TS. The prevalence and relationship between periodontal disease and pre-term low birth weight infants at King Khalid University Hospital in Riyadh, Saudi Arabia. J Contemp Dent Pract 2004; 5: 40-56.

85. Mojon P, Budtz-Jorgensen E, Rapin CH. Relationship between oral health and nutrition in very old people. Age Ageing 1999; 28:463-8.

86. Abnet CC, Qiao YL, Mark SD, Dong ZW, Taylor PR, Dawsey SM. Prospective study of tooth loss and incident esophageal and gastric cancers in China. Cancer Causes Control 2001; 12:847-54.

87. Abnet CC, Kamangar F, Dawsey SM, StolzenbergSolomon RZ, Albanes D, Pietinen P, et al. Tooth loss is associated with increased risk of gastric non-cardia adenocarcinoma in a cohort of Finnish smokers. Scand J Gastroenterol 2005; 40:681-7.

88. Wei WQ, Abnet CC, Lu N, Roth MJ, Wang GQ, Dye BA et al. Risk factors for oesophageal squamous dysplasia in adult inhabitants of a high risk region of China. Gut 2005; 54:759-63.

89. Sepehr A, Kamangar F, Fahimi S, Saidi F, Abnet CC, Dawsey SM. Poor oral health as a risk factor for esophageal squamous dysplasia in northeastern Iran. Anticancer Res 2005; 25:543-6.

\section{Revista Biomédica}




\section{Políticas de salud bucal en México}

90. Alvarez-Manilla JM. Atención primaria a la salud. Salud Publica Mex 1988; 30:678-82.

91. World Health Organization. Declaration of Alma-Ata International Conference on Primary Health Care, AlmaAta, USSR, 6-12 September 1978. Disponible en: http: //www.who.int/hpr/NPH/docs/declaration_almaata.pdf consultado [agosto-2004].

92. WHO/FDI. Global goals for oral health in the year 2000. Int Dent J 1982; 32:74-7.

93. World Health Organization. The World Oral Health Report 2003. Continuous improvement of oral health in the 21st century - the approach of the WHO Global Oral Health Programme. WHO Geneva; 2003.

94. Secretaría de Salud. Programa Nacional de Salud 20012006. Estrategia: reducir los rezagos en salud que afectan a los pobres. Programa de Acción: Salud Bucal. SSA México; 2001.

95. Bratthall D. Introducing the Significant Caries Index together with a proposal for a new global oral health goal for 12-year-olds. Int Dent J 2000; 50:378-84.

96. Hobdell M, Petersen PE, Clarkson J, Johnson N. Global goals for oral health 2020. Int Dent J 2003; 53:285-8.

97. Secretaría de Comercio y Fomento Industrial. Norma Oficial Mexicana NOM F-8-1988. Alimentos, Sal Yodada y Sal Fluorudada. SECOFI; 1988.

98. Secretaría de Salud. Reglamento de la ley general de salud en materia de control sanitario de actividades, establecimientos, productos y servicios. SSA México; 1988.

99. Secretaría de Salud. Reglamento de yodación y fluoruración de la sal. D.O.F. México DF. 1981.

100. SSA, SECOFI, AMISAC. Acuerdo de concertación para la fluoruración-yodación de la sal en México. 1991.

101. Secretaría de Salud. Dirección General de Medicina Preventiva. Dirección de Enfermedades Crónico Degenerativas. Subdirección de Prevención y Control de Enfermedades Bucales. Lineamientos para la dosificación de fluoruro sistémico. Bases fisiológicas. SSA México; 1992.

102. Secretaría de Salud. Subsecretaría de Prevención y control de enfermedades. Programa de Salud Bucal. SSA México; 2000.
103. Secretaría de Salud. Norma Oficial Mexicana NOM009-SSA2-1993, Para el Fomento de la Salud del Escolar. SSA México; 1994.

104. Secretaría de Salud. Informe de labores 1988-1989. SSA; 1989.

105. Secretaría de salud. Subsecretaría de Servicios de Salud. Dirección General de Medicina Preventiva. Programa de trabajo 1994. Grupo interinstitucional de salud bucal. SSA México; 1994.

106. Comité Interinstitucional de Odontología. Programa nacional educativo preventivo contra caries y parodontopatías en preescolares y escolares. SSA, IMSS, ISSSTE, DIF, SEP, ADM; 1990.

107. Secretaría de Salud. Norma Oficial Mexicana NOM-013-SSA2-1994, Para la prevención y control de enfermedades bucales. SSA México; 1995.

108. Secretaría de Salud. MODIFICACION a la Norma Oficial Mexicana NOM-013-SSA2-1994, Para la prevención y control de enfermedades bucales, publicada el 6 de enero de 1995. SSA México; 1999.

109. Secretaría de Salud. Norma Oficial Mexicana NOM040-SSA1-1993, Bienes y Servicios. Sal Yodada y Sal Yodada Fluorurada. Especificaciones Sanitarias. SSA México; 1995.

110. Presidencia de la República. Plan Nacional de Desarrollo 2001-2006. Presidencia de la República de México. 2001.

111. Diario Oficial de la Federación. DECRETO por el que se establece el Sistema de Cartillas Nacionales de Salud. Diario Oficial de la Federación 24-Diciembre-2003. Disponible en: http://www.salud.gob.mx/unidades/dgps/ cartillas/doctos_descarga/DOF-24-DIC-02.doc Consultado [Agosto 2 de 2004].

112. Centers for Disease Control and Prevention. Recommendations for using fluoride to prevent and control dental caries in the United States. MMWR 2001;50(No. RR-14).

113. Barrandey OSE, Cabello AMV, Magaña RJ, Rodríguez DE. Sal fluorada, riesgo o beneficio para la población de la Ciudad de Chihuahua. Rev ADM 1994; 51:80-9.

114. Loyola-Rodríguez JP, Pozos-Guillen AJ, HernándezGuerrero JC. Bebidas embotelladas como fuentes adicionales de exposición a fluor. Salud Publica Mex 1998; 40:438-41. 


\section{CE Medina-Solis, G Maupomé, L Avila-Burgos, R Pérez-Núñez, B Pelcastre-Villafuerte, y col.}

115. Loyola-Rodríguez JP, Pozos-Guillén AJ, RuedaGonzález AM, Vázquez-Moctezuma S, de la Paz-Domínguez G. Factores de riesgo de fluorosis dental en San Luis Potosí, México. Rev ADM 1996; 53:295-300.

116. Diaz-Barriga F, Leyva R, Quistian J, Loyola-Rodriguez JP, Pozos A, Grimaldo M. Endemic fluorosis in San Luis Potosi, Mexico. IV. Sources of fluoride exposure. Fluoride 1997; 30:219-22.

117. Diaz-Barriga F, Navarro-Quezada A, Grijalva M, Grimaldo M, Loyola-Rodriguez JP, Deogracias M. Endemic fluorosis in México. Fluoride 1997; 30:223-39.

118. Alarcón-Herrera MT, Martín-Domínguez IR, TrejoVázquez R, Rodríguez-Dozal S. Well water fluoride, dental fluorosis, and bone fractures in the Guadiana Valley of Mexico. Fluoride 2001; 34:139-49.

119. Lalumandier JA, Rozier G. Parent's satisfaction with children's tooth color: fluorosis as a contributing factor. J Am Dent Assoc 1998; 129:1000-6.

120. Burt BA, Keels MA, Heller KE. The effects of break in water fluoridation on the development of dental caries and fluorosis. J Dent Res 2000; 79:761-9.

121. Martinez-Mier EA, Soto-Rojas AE, Ureña-Cirett JL, Katz BP, Stookey GK, Dunipace AJ. Dental fluorosis and altitude: a pilot study. Oral Health Prev Dent 2004; 2:3948.

122. Shulman JD, Maupomé G, Clark DC, Levy SM. Perseptions of desirable tooth color among parents, dentisits and children. J Am Dent Assoc 2004; 135:595-604.

123. Soto Rojas AE, Ureña-Cirett JL, Martínez-Mier EA. A review of the prevalence of dental fluorosis in Mexico. Pan Am J Public Health 2004; 15:9-17.

124. Rodríguez-Dozal S, Alarcón Herrera MT, Cifuentes E, Barraza A, Loyola Rodríguez JP, Sanin LH. Dental fluorosis in rural communities of Chihuahua, Mexico. Fluoride 2005; 38:125-32.

125. Beltrán-Valladares PR, Cocom-Tum H, CasanovaRosado JF, Vallejos-Sánchez AA, Medina-Solís CE, Maupomé G. Prevalencia de fluorosis dental y fuentes adicionales de exposición a fluoruro como factores de riesgo a fluorosis dental en escolares de Campeche, México. Rev Invest Clin 2005; 57:532-9.
126. Vallejos-Sánchez AA, Medina-Solís CE, CasanovaRosado JF, Maupomé G, Minaya-Sánchez M, Pérez-Olivares $\mathrm{S}$. Dental fluorosis in cohorts born before, during and after the national salt fluoridation program in a community in Mexico. Acta Odontol Scand 2006; 64:209-13.

127. Maupomé G, Diez-de-Bonilla J, López R. La educación dental en un momento crítico. Consideraciones generales en el entorno profesional de América del Norte. Salud Pública Mex 1997; 39:554-64.

128. Maupomé G. Preparación de los dentistas de mañana, de acuerdo a las necesidades de hoy. Salud Pública Mex 2000; 42:178-80.

129. Maupomé G, Ramírez-Mireles LE. Why a School Dental Service is necessary. Part I. The Human resources and dental morbidity horizon. Pract Odontol 2001; 22:6-12.

130. Maupomé G, Ramírez-Mireles LE. Why a School Dental Service is necessary. Part II. Considerations about a health policy. Pract Odontol 2001; 22:31-5.

131. Maupomé G. Who is Filling What.: The contrast between the oral health situation and human health resources in Mexico. Critical Public Health 2000; 10:153-66.

132. Medina-Solís CE, Casanova-Rosado AJ, CasanovaRosado JF, Vallejos-Sánchez AA, Maupomé G, Ávila-Burgos L. Factores socioeconómicos y dentales asociados a la utilización de servicios dentales en escolares de Campeche, México. Bol Med Hosp Infant Mex 2004; 61:324-33.

133. Medina-Solís CE, Maupomé G, Ávila-Burgos L, Casanova-Rosado JF, Vallejos-Sánchez AA, SegoviaVillanueva A. Utilización de servicios odontológicos de salud por niños menores de 5 años con seguridad social. Rev Mex Pediatr 2004; 71:222-8.

134. Medina-Solís CE, Maupomé G, Avila-Burgos L, HijarMedina M, Segovia-Villanueva A. Pérez-Núñez R. Factors that modify the use of dental services in Mexican preschool children. Pediatr Dent 2006; 28:285-92.

135. Pérez-Núñez R, Medina-Solis CE, Maupomé G, Vargas-Palacios A. Factors associated with dental health care coverage in Mexico: Findings from the National Performance Evaluation Survey 2002-2003. Community Dent Oral Epidemiol 2006; 34:387-97.

\section{Revista Biomédica}

